\title{
A COMPARATIVE STUDY BETWEEN THE OUTCOME OF LAPAROSCOPIC APPENDECTOMY AND OPEN APPENDECTOMY IN CHILDREN
}

\author{
NS FERDOUS ${ }^{1}$, A HUQ $^{2}, \mathrm{~K} \mathrm{HASINA}{ }^{3}, \mathrm{M} \mathrm{KHATUN}^{4}$
}

\begin{abstract}
:
Background: Laparoscopic surgery for acute appendicitis in children has been proposed to have advantages over conventional surgery.

Introduction: Appendicitis is the most common surgical emergency in childhood. ${ }^{1}$ For decades, Open appendectomy (OA) has been the standard treatment for all forms of appendicitis with excellent results. ${ }^{2}$ Since its description in the early 1980s, laparoscopic appendectomy (LA) became an acceptable approach for simple appendicitis in children. ${ }^{4,}{ }^{5}$ However, its role in the treatment of complicated appendicitis is controversial. Today, in developed countries about $8 \%$ of the population is appendectomized for acute appendicitis during their lifetime. ${ }^{7}$

Materials and method: We are conducting a prospective comparative study with the intention to observe the outcome of 60 purposively selected patients of acute appendicitis in the Department of Pediatric Surgery, Dhaka Medical College \& Hospital (DMCH), Dhaka, over a period of 12 months from October, 2013 to October, 2014. The patients are going to be divided into 2 groups by random sampling- Group A- OA Group (patients underwent open appendectomy) and Group B- LA Group (patients underwent laparoscopic appendectomy) for uncomplicated appendicitis. Children up to 14 years are planned to be selected as study subjects. All children are subjected to investigate for white blood cell (WBC) count, plain X- ray KUB region, urine R/E, preoperatively and to observe the amount of analgesics in postoperative pain management and cosmesis on first week, first month, and third month of operation. Structured questionnaire is used to collect information regarding pain, fever, other association preoperatively and operative procedure, duration of operation, per operative findings and post operative follow up. Informed written consent from parents or legal guardian is taken after describing the study objectives. Ethical clearance has been sought from the Ethical Committee of Dhaka Medical College.

Results: Total 23 patients are studied till now, 16 in LA group and 7 in OA group. From this limited data we have seen that there is apparently minimum difference between two groups of study population regarding postoperative outcomes.

Conclusion: This is an on-going study. Definite conclusion could not be drawn at this preliminary stage. Laparoscopic appendectomy for uncomplicated appendicitis in children is feasible and safe. It is associated with a significantly less use of post operative pain killer, lower incidence of wound infection, and reduced length of hospital stay when compared with patients who had open appendectomy.
\end{abstract}

Key words: uncomplicated appendicitis, laparoscopic and open appendectomy, post operative analgesics, cosmesis

1. Dr. Nazmus Sakib Ferdous, MS - thesis part student, Department of Pediatric Surgery, Dhaka Medical College \& Hospital, Dhaka.

2. Dr. Ashraf-Ul-Huq Professor and Head, Department of Pediatric Surgery, Dhaka Medical College \& Hospital, Dhaka

3. Dr. Kaniz Hasina, Associate Professor, Department of Pediatric Surgery, Dhaka Medical College \& Hospital, Dhaka.

4. Dr. Masuda Khatun, Assistant Professor, Department of Dermatology, Shaheed Suhrawardy Medical College \& Hospital.

Correspondence to: Dr. Nazmus Sakib Ferdous, MS - thesis part student, Department of Pediatric Surgery, Dhaka Medical College \& Hospital, Dhaka. e-mail: ferdousn3@gmail.com

\section{Introduction:}

Appendicitis is the most common surgical emergency in childhood. ${ }^{1}$ Appendicitis was first recognized as a disease entity in the sixteenth century and was called perityphlitis. McBurney described the clinical findings in 1889.

For decades, Open appendectomy has been the standard treatment for all forms of appendicitis with excellent results. ${ }^{2}$ 
Since its description in the early 1980 s, laparoscopic appendectomy became an acceptable approach for simple appendicitis in children. ${ }^{3,4,5}$ However; its role in the treatment of complicated appendicitis is controversial.

Since its introduction by McBurney in 1894 appendectomy is the treatment of choice for acute appendicitis. ${ }^{6}$ It soon became one of the most frequently performed surgical procedures. Today, in developed countries about $8 \%$ of the population is appendectomized for acute appendicitis during their lifetime. $^{7}$

The surgical technique remained nearly unchanged for over a century, as it combines therapeutic efficacy with low morbidity and mortality rates. ${ }^{8}$ The evolution of endoscopic surgery led to the idea of performing appendectomy in laparoscopy, which was first described by Semm in $1983 .{ }^{9}$ Nevertheless, the new method has only partly gained acceptance ${ }^{10}$, because the advantages of LA were not as obvious as for laparoscopic cholecystectomy, while some studies claimed laparoscopic appendectomy to be superior to $O A$ in terms of a quicker and less painful recovery, less postoperative complications, and better cosmesis, other studies found no such advantages or even favored the traditional approach. Beside these therapeutic effects of LA, laparoscopy per se may offer valuable diagnostic opportunities.

Since surgical removal of an uninflamed, normal ('innocent') appendix occurs in up to $50 \%$ of patients, it has been proposed not to remove the appendix in those situations, where other pathologies can be diagnosed during laparoscopy. Some surgeons therefore have used appendix laparoscopy as a diagnostic tool only, and perform conventional appendectomy after laparoscopy in those patients, where the macroscopically has an abnormal appearance. However, it is not yet clarified, in which situations a normal looking appendix should be left in place, although non-randomized studies indicate this. ${ }^{11}$

The large number of published trials and the still ongoing debate prompted us to undertake a systematic review of all randomized trials which compared therapeutic and diagnostic advantages of both techniques.

The large number of published trials and the still ongoing debate prompted us to undertake a systematic review of all randomized trials which compared therapeutic and diagnostic advantages of both techniques.

Laparoscopic appendectomy is being done at a time when laparoscopic cholecystectomy has shown definite benefits over the open technique. In the young female the cause of lower abdominal pain is often gynaecological. Gynaecologists perform diagnostic laparoscopy frequently. Semm, a German gynaecologist, performed the first laparoscopic appendectomy in 1983.

Laparoscopic cholecystectomy is now the gold standard for cholelithiasis and has virtually replaced open cholecystectomy. However, is this case for acute appendicitis? The role of

laparoscopic appendectomy has not yet been clearly defined. Laparoscopic surgery continues to evolve at such a rapid pace that it is now time to examine the latest developments with regard to acute appendicitis. Numerous factors need to be considered in deciding the ideal, and most appropriate surgical technique for acute appendicitis.

Although laparoscopic appendectomy is gaining popularity, open appendectomy has remained popular with surgeons caring for children. The reasons for this include the increased skill level necessary for pediatric laparoscopic procedures, concerns over increased operating times and costs, and fears that the laparoscopic approach to appendicitis is somehow associated with an increased complication rate.

There is a group of surgeons who are advocating laparoscopic appendectomy in all cases of appendicitis in pediatric patients. In one prospective non randomized trial 500 appendectomies

were studied, 362 children underwent open procedure and 138 underwent laparoscopic appendectomy. There was no mortality in either group. Major complications were $3 \%$ in open

group but no major complications were seen in the laparoscopic group. Minor complications were $20 \%$ in open and $13 \%$ in laparoscopic appendectomy. ${ }^{12}$ Paya et al published a prospective study of 75 children with perforated appendicitis. Ten underwent laparoscopic appendectomy and the remainder underwent open operation. There were no postoperative abscesses in the laparoscopic group but $2(3.1 \%)$ of 65 patients who had open appendectomies developed postoperative intra-abdominal abscesses. ${ }^{13}$ 
In a prospective series of children aged 4-12 years, reported from Cairo, 48 underwent open appendectomy and 34 laparoscopic operation, over a 6 month period.. Wound complications were fewer, cosmetic appearance better, and time to return to normal activities quicker ( 7 cf 12 days) in the laparoscopic group. ${ }^{13}$ Lintula $\mathrm{H}$. et al studied the effect of laparoscopic appendectomy in children between 4$15 \mathrm{yr}$ of age and demonstrated that laparoscopic appendectomy was not associated with any increase risk of intra-operative or long term complications. ${ }^{14}$

\section{Advantages of laparoscopic method:}

Shorter hospital stay

Faster recovery

Least post operative pain

Fewer post operative complications

Minimal scarring

\section{Extra-Appendicular Findings at Laparoscopy:}

Ovarian cyst

Meckel's diverticulum

Torsion of the appendix epiploicae

Adhesions

Intestinal rotation anomaly

Hydatid cyst

The disadvantages include risks of injury to abdominal organs from trocar or instrument insertion, which are greatly reduced when laparoscopy is performed by experienced surgeons.

\section{Materials and Method:}

We are conducting a prospective comparative study with the intention to observe the postoperative outcome of 60 purposively selected patients of appendectomy in the Department of Pediatric surgery, Dhaka Medical College Hospital (DMCH), Dhaka, over a period of 12 months from October, 2013 to October, 2014. The patients are going to be divided into 2 groups by random sampling-Group A (patients underwent open appendectomy) and Group B (patients underwent laparoscopic appendectomy) for uncomplicated appendicitis .Children up to 14 years are planned to be selected as study subjects. All children are subjected to investigate for white blood cell (WBC) count, plain x-ray KUB region, urine $R / E$, preoperatively and amount of analgesics in postoperative pain management and cosmesis on $1^{\text {st }}$ week, $1^{\text {st }}$ month, and 3rd month of operation. Structured questionnaire is used to collect information regarding pain, fever, other association preoperatively and operative procedure, duration of operation, per operative findings, post operative complication and follow up. Informed written consent from parents or legal guardian is taken after describing the study objectives. Ethical clearance has been sought from the Ethical Committee of Dhaka Medical College. Data collected includes demographic, duration of symptoms, operative time, analgesia, complications, and length of hospitalization. Laparoscopic appendectomy was done by three trocar technique in all cases. Inclusion Criteria features of acute appendicitis with age group up to 14 years of age. Exclusion Criteria, Patients were excluded if the diagnosis of appendicitis was not clinically established. Features of complicated appendicitis, patients with co morbid conditions, Age : $>14$ years.Non consenting patients are excluded from the study.

\section{Results:}

Total 23 patients are studied till now, 16 in laparoscopic group (Group B) and 7 in open group (Group A). Out of the 23 patients 7 were female and 16 male. In male 12 were in age group of (4-9) years old and 10 were (10-14) years. The average operating time was 60 minutes for LA and 30 minutes for the OA group. Mean hospital stay was 3 days and 4 days for LA and OA group respectively. The post operative narcotic analgesic requirement was minimal in LA group. In open appendectomy one out of 3 patients developed superficial wound infection. There was no post operative intra-abdominal collection in laparoscopic appendectomy. There was no conversion patient in the LA patients. There was no mortality in the current series.

\section{Discussion:}

The overall objective of this study is to compare the safety and benefits of laparoscopic versus open appendectomy in child. To detect and compare the amount of analgesics for post operative pain and wound appearance score of post operative scar in laparoscopic appendectomy and open appendectomy in children. Our hypothesis was to compare that the outcome of laparoscopic appendectomy is superior to open appendectomy in Children.

In this study comparing the length of hospital stay, postoperative pain control, hospital morbidity and mortality, and rate of routine discharge in patients 
undergoing LA and OA based on data from administrative database. In our investigation patients undergoing $L A$ had a significantly shorter median length of hospital stay (LA: 4 days, OA: 3 days,) and higher rate of routine discharge compared with OA patients. Patients after LA had significantly less amount of analgesics are used in control of postoperative pain, less infections gastrointestinal complications and overall complications.

The question of whether LA decreases the length of hospitalization has been a matter of great debate over the past decade. ${ }^{15,16}$ Some randomized controlled trials associated LA with decreased hospital stay; however, others report no significant difference between LA and OA.

Most retrospective chart reviews, randomized controlled trials, and meta-analyses report similar occurrence in overall postoperative morbidity for LA and $\mathrm{OA},{ }^{17}$ whereas only a few investigations found statistically significant differences. Some investigations found significantly higher postoperative wound infections after OA, whereas others reported similar rates. In a recent meta-analysis, Golub and colleagues found a wound infection rate for LA that was less than half the rate in patients undergoing OA. Conversely, the authors reported an increase in the rate of intraabdominal abscesses after $L A$, which failed, however, to reach statistical significance. Other meta-analyses confirm these findings. Although it can be assumed that the definition of intraabdominal abscess is uniform among different studies, wound infection can be defined with great variability ranging from slight erythema to purulent secretion. ${ }^{18}$ This variability strongly influences the rate of reported postoperative wound infections. In the present investigation no postoperative infections is seen in LA. This is a drawback of our investigation for which we could not adjust. Besides overall complication rate and postoperative infections, the occurrence of gastrointestinal complications was significantly rarer in LA patients, whereas no difference could be found for pulmonary, urinary, systemic, mechanical wound, and intraoperative complications. Only few studies report pulmonary, gastrointestinal, and urinary complications. ${ }^{19}$ None of these studies found, however, a significant difference between LA and OA.

In most of randomized clinical trials and chart reviews comparing LA versus $O A$, no mortality was reported in either group. This is to be expected because appendicitis is a disease that disproportionally strikes young, healthy people, and appendectomy is a lowrisk surgical procedure.

In the present investigation routine discharge was significantly higher in patients undergoing $L A$ versus $O A$. Patients after LA were 3 times more likely to be discharged routinely compared with OA patients. To our knowledge, no other study has compared the rate of routine discharge between LA and OA. Some investigations have, however, assessed the impact of the surgical procedure on return to normal activities. Several studies found LA to be associated with significantly earlier return to normal activities compared with OA. ${ }^{20}$

\section{Conclusion:}

This is an on-going study. Definite conclusion could not be drawn at this preliminary stage. Laparoscopic appendectomy is equally safe and can provide less post operative morbidity in experienced hands, as in open appendectomy. Most cases of acute appendicitis can treated laparoscopically. Laparoscopic appendectomy is useful method for reducing hospital stay, complications and return to normal activity. With better training minimal access surgery is now available. The time has arrived for it to take its place in the surgeon's repertoire. In summary, we have shown that LA has significant advantages over OA with respect to post operative pain management, length of hospital stay, rate of routine discharge, postoperative in-hospital morbidity and cosmoses. Our findings may have important health care implications, not only resulting in clinical patient benefit, but also lowering hospital costs. Exponentially increasing health costs have stimulated a massive health care reform effort, seeking cost containment. It is imperative that healthcare professionals make fiscally prudent decisions, as the present environment necessitates a critical appraisal of apparently equi-efficacious therapeutic modalities. However, all aspects of LA and OA must be compared, including postoperative pain, patient's quality of life, and days away from daily activities, procedural costs, total costs, and long-term complications. Further analyses to evaluate the abovementioned endpoints are required to define whether LA should be considered the treatment of choice for uncomplicated appendicitis in children.

\section{References:}

1. Gilchrist BF, Lobe TE, Schropp KP et al. Is there a role for laparoscopic appendectomy in pediatric surgery. J Pediatr Surg 1992, 27: 209-214. 
2. Pearl RH, Hale DA, Molloy $M$ et al. Pediatric appendectomy. J Pediatr. Surg 1995, 30: 173-181.

3. Semm K: Endoscopic Appendectomy. Endosc.1992,15: 59-64.

4. Attwood SEA, Hill ADIL, Murphy PG et al. A prospective randomized trial of laparoscopic versus open appendectomy. Surg 1992, 112: 497-501.

5. Kim CK, Hgoi SS, Goh PMY et al : Randomized controlled trial comparing laparoscopic and open appendectomy. Br J Surg PO: 1993,1599-1600.

6. McBurney $\mathrm{C}$. The incision made in the abdominal wall in cases of appendicitis, with a description of a new method of operating .Ann Surg 1894; 20:38.

7. Addiss DG, Shaffer N, Fowler BS, Tauxe RV. Theepidemiology of appendicitis and appendectomy in theUnited States. Am J Epidemiol 1990;132:910-25.

8. Eriksson S, Granström L. Randomized controlled trial of appendicectomy versus antibiotic therapy for acute appendicitis. Br J Surg 1995;82: $166-9$.

9. Semm K. Endoscopic appendectomy. Endoscopy 1983;15: 59-64.

10. Faiz O, Clark J, Brown T, Bottle A, Antoniou A,Farrands P, Darzi A, Aylin P. Traditional and laparoscopic appendectomy in adults: outcomes in English NHS hospitals between 1996 and 2006. Ann Surg 2008;248: 800-6.

11. Van den Broek WT, Bijnen AB, De Ruiter P, Gouma DJ. A normal appendix found during diagnostic laparoscopy should not be removed. Br J Surg 2001;88:251-4.

12. Paya .k.Fakhari M.Rouhofer Felberbaue FX Rebhandi W.Horcher E.JSLS J.Soc lapaoendos Surgery 2000, $4.121-24$
13. Paya K.Rouhofer U Rebhandi W. Deluggi St Horcher E.Perforating appendicitis : an indication for appendicitis? Surg Endos 2000:182-84

14. Lintula .H. Kokki H. Vannamo .k.single blind randomized control trial of Laparoscopc versus open appendectomy in children.Br.S.Surg $2001(88) 4: 510-4$

15. Fingerhut $A$, Millat $B$, Borrie F. Laparoscopic versus open appendectomy: time to decide. World J Surg. 1999;23:835-845.

17. Temple LK, Litwin DE, McLeod RS. A metaanalysis of laparoscopic versus open appendectomy in patients suspected of having acute appendicitis. Can J Surg. 1999;42: 377-383.

3. Editorial. A sound approach to the diagnosis of acute appendicitis. Lancet. 1987;i:198-200.

6. Maxwell JG, Robinson CL, Maxwell TG, et al. Deriving the indications for laparoscopic appendectomy from a comparison of the outcomes of laparoscopic and open appendectomy. Am J Surg. 2001;182:687-692.

8. Fingerhut $A$, Millat $B$, Borrie F. Laparoscopic versus open appendectomy: time to decide. World J Surg. 1999;23:835-845.

11. Charlson ME, Pompei P, Ales KL, et al. A new method of classifying prognostic comorbidity in longitudinal studies: development and validation. J Chronic Dis. 1987;40:373-383.

17. Temple LK, Litwin DE, McLeod RS. A metaanalysis of laparoscopic versus open appendectomy in patients suspected of having acute appendicitis. Can J Surg. 1999;42:377383.

19. Nazzal M, Ali MA, Turfah F, et al. Laparoscopic appendectomy: a viable alternative approach. $J$ Laparoendosc Adv Surg Tech A. 1997;7:1-6.

20. Heinzelmann M, Simmen HP, Cummins AS, et al. Is laparoscopic appendectomy the new 'gold standard' Arch Surg. 1995;130:782-785. 